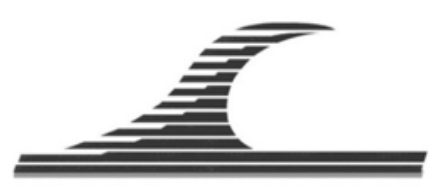

REVUE PARALIA

\title{
La bioréceptivité de matériaux cimentaires dans l'eau de mer : mécanismes, facteurs agissants et conséquences
}

\author{
Mahmoud HAYEK ${ }^{1}$, Marie SALGUES ${ }^{1}$, Frédéric HABOUZIT ${ }^{2}$, \\ Sandrine BAYLE ${ }^{3}$, Jean-Claude SOUCHE ${ }^{1}$, \\ Klartjee DE WEERDT ${ }^{4}$, Sylvain PIOCH ${ }^{5}$
}

1. LMGC, IMT Mines Alès, Univ. Montpellier, CNRS, 6, avenue de Clavières, 30319 Alès Cedex, France.

Mahmoud.hayek@mines-ales.fr ;marie.salgues@mines-ales.fr ; jean-claude.souche@mines-ales.fr

2. INRA, UR0050, Laboratoire de Biotechnologie de l'Environnement (LBE), 102 Avenue des Étangs, 11100 Narbonne, France.

frederic.habouzit@inra.fr

3. IMT Mines Alès, Laboratoire de génie de l'environnement industriel (LGEI), 6, avenue de Clavières, 30319 Alès Cedex, France.

sandrine.bayle@mines-ales.fr

4. Norwegian University of Science and Technology NTNU, Department of Structural Engineering, Trondheim, Norway.

klaartje.d.weerdt@ntnu.no

5. UMR 5175 CEFE, Université Paul Valéry, Montpellier, SupAgro, EPHE, Laboratoire Biologie Écologie Environnement - UPV, Route de Mende, 34000 Montpellier, France.

sylvain.pioch@univ-montp3.fr

\section{Résumé :}

Le béton est un matériau composite complexe, dont les différents composants lui permettent de s'adapter à des usages très différents grâce à ses caractéristiques physicochimiques adaptables à des cahiers des charges très variés. Ses performances mécaniques, sa durabilité exceptionnelle et son coût modéré justifient l'utilisation large de ce matériau dans la construction des ouvrages maritimes. Immergé dans l'environnement marin, le béton est susceptible d'être colonisé par les différents organismes marins qui se regroupent ensemble sur la surface sous leur forme sessile nommée biofilm/biofouling. Cette colonisation forme le point de départ de différentes interactions biologiques pouvant avoir lieu entre le matériau et son environnement marin (biodéterioration/bioprotection). 
Le cycle et la vitesse de colonisation vont dépendre des conditions environnementales comme de la nature du substrat (composition, propriétés physico-chimiques du support...) ainsi que des propriétés de microorganismes concernés. Dans le but d'étudier la bioréceptivité du béton dans l'environnement marin, cet article se focalise successivement sur les paramètres du milieu marin qui influent sur sa colonisation et sur les organismes concernés. Les caractéristiques intrinsèques du béton qui sont susceptibles d'influencer la bioréceptivité et les mécanismes conduisant à la formation du biofilm et à la biocolonisation des bétons sont décrits. Enfin, le sujet de l'effet positif (bioprotection) ou négatif (biodéterioration) des organismes vivants sur le béton est évoqué.

\title{
Bioreceptivity of cementitious materials in seawater: mechanisms, acting factors and consequences
}

\begin{abstract}
:
Concrete is a complex composite material; whose different components allow it to adapt to very different uses due to its physicochemical characteristics adaptable to very varied conditions. Its mechanical performance, exceptional durability and moderate cost explain the wide use of this material in the construction of marine structures. Submerged in the marine environment, the concrete will be colonized by various marine organisms that gather together on the surface in their sessile form called biofilm/biofouling. This colonization is the starting point for different biological interactions that may occur between the material and its marine environment (biodeterioration/bioprotection). The cycle and the rate of colonization will depend on environmental conditions such as the nature of the substrate (composition, physicochemical properties of the support, etc.) as well as the properties of the concerned microorganisms. In order to study the bioreceptivity of concrete in the marine environment, this paper focuses successively on the characteristics of the marine environment that affect the colonization of the concrete and the concerned organisms. Then, the intrinsic parameters of concrete that can influence bioreceptivity are listed and described. The same goes for the mechanisms leading to the biofilm formation and the biocolonisation of concretes. Finally, the subject of the positive (bioprotection) or negative (biodeterioration) effect of the biofilm formation on concrete is discussed.
\end{abstract}

Key words:

Concrete, Marine environment, Biocolonization, Biofilm/Biofouling, Bioreceptivity/Biodeterioration. 


\section{Introduction}

Avec $190 \mathrm{~m}^{3}$ coulés chaque seconde, soit 6 milliards de $\mathrm{m}^{3}$ par an, le béton est le matériau manufacturé le plus utilisé au monde (PLANETOSCOPE, 2020) du fait de sa durabilité, sa résistance et son faible coût. Les ouvrages en béton en milieu maritime sont présents sous de multiples formes d'aménagements portuaires et côtiers destinés à des fonctionnalités multiples. Le tableau 1 rappelle les principaux types d'ouvrages en sites maritimes et côtiers. Le tableau 1 est limité aux ouvrages les plus courants pour lesquels la colonisation par le biofilm et le biofouling peut représenter un intérêt et font l'objet d'études citées plus loin dans l'article.

Ils constituent un support pour les organismes marins, peuvent favoriser le développement d'écosystèmes diversifiés et ainsi améliorer la biodiversité marine. La biocolonisation des bétons en milieu marin suscite l'attention des scientifiques ces dernières années, mais peu d'études sont réalisées en comparaison avec celles relatives avec leur résistance et leur durabilité.

En effet, l'environnement marin est considéré comme un milieu extrêmement agressif pour les ouvrages en béton. L'action de la houle et des vagues, ainsi que les efforts importants dus aux actions d'accostage et d'amarrage des navires représentent des agressions érosives et mécaniques importantes sur les ouvrages.

Les actions physico-chimiques sont aussi à considérer compte tenu de la composition de l'eau de mer. L'eau de mer contient approximativement entre 30 à $40 \mathrm{~g} / \mathrm{L}$ de sels dissous (NGUYEN, 2005). Les ions majoritaires sont: $\mathrm{Cl}^{-}, \mathrm{SO}_{4}{ }^{2-}, \mathrm{Br}^{-}, \mathrm{Na}^{+}, \mathrm{Mg}^{2+}, \mathrm{Ca}^{2+}$ et $\mathrm{K}^{+}$ (VICHOT \& OLLIVIER, 2008). La composition de l'eau de mer reste relativement constante et les eaux provenant de différents océans et mers ne diffèrent que faiblement (SIGG et al., 2001). Les agressions chimiques des bétons causées par les ions contenus dans l'eau de mer sont nombreuses et peuvent conduire à des détériorations des ouvrages (SOSA et al., 2011).

Contrairement aux actions mécaniques ou physico-chimiques qui sont bien appréhendées, documentées et normalisées pour optimiser la résistance et la durabilité des matériaux cimentaires, celles de nature biologique sont beaucoup moins connues et généralement négligées. Dans la perception de l'ingénieur en génie civil maritime, l'ouvrage immergé est imaginé comme un ouvrage terrestre avec une surface de béton lisse peu évolutive dans le temps. Quant à la colonisation par les organismes vivants, si elle est constatée, elle n'amène pas de questionnement particulier sauf pour les ouvrages flottants où l'ingénieur s'est rendu compte que la biocolonisation influait sur le tirant d'eau et le comportement en flottaison.

Les études concernant la détérioration biologique des bétons et leurs aptitudes à être colonisés par des microorganismes se sont donc intensifiées ces dernières années. Dans ce contexte, la bioréceptivité d'un matériau cimentaire a été définie comme l'aptitude du matériau à être colonisé par un ou plusieurs groupes d'organismes vivants. Cette bioréceptivité est gouvernée par l'interaction de trois acteurs (Figure 1) : le matériau en 
question (l'état et la nature de la surface, la composition chimique et les propriétés physico-chimiques), les microorganismes vivants (la nature de microorganisme, l'hydrophobicité de sa surface, la présence de flagelle et la nature de la matrice secrétée) et le milieu environnant (l'alcalinité du milieu, la disponibilité de nutriments, la température et la lumière) dans lesquels se trouvent les deux premiers.

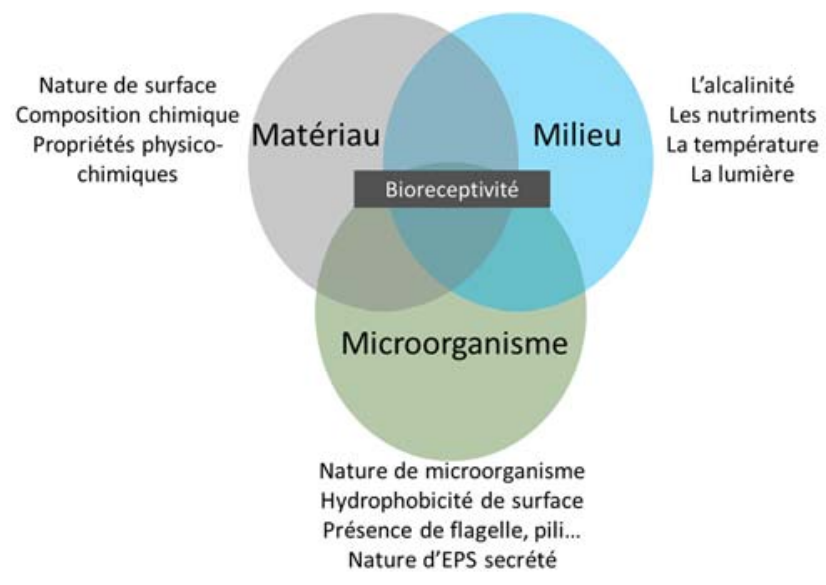

Figure 1. Les facteurs qui influencent la bioréceptivité de matériaux cimentaires.

Dans l'environnement marin, toute surface immergée dans l'eau de mer est susceptible d'être colonisée par les différents microorganismes marins qui adhérent presque instantanément à cette surface. Ces microorganismes vont croître et se développer pour former à terme un biofilm (LORS et al., 2017). Cette colonisation biologique forme le point de départ des différentes interactions biologiques qui ont lieu entre le matériau et l'environnement marin.

La détérioration des matériaux cimentaires par l'action de microorganismes vivants marins est un phénomène d'ores et déjà établi (VISHWAKARMA et al., 2014) dont différentes formes d'actions d'origine biologique peuvent être observées, telles que la biodétérioration esthétique, mécanique ou chimique (LORS et al., 2017).

Les microorganismes marins (bactéries, algues, champignons...) colonisent la surface immergée ou humide (zone de marnage) et interagissent avec le matériau support (SALTA et al., 2013).

Une biodétérioration esthétique peut alors modifier l'aspect visuel du matériau (apparition de tâches et de salissures colorées) sans remettre en cause sa structure ni sa durabilité (TRAN et al., 2014). Toutefois, les performances peuvent être réduites lors de la pénétration des microalgues dans la porosité ouverte du matériau et sous l'action des acides organiques produits par les activités métaboliques des algues. Ces acides qui agissent avec les matériaux cimentaires amènent à une perte de la résistance mécanique et par suite à une biodéterioration du matériau (SILVA and NAIK, 2013). 
Néanmoins, certaines études récentes montrent une bioprotection du béton lorsqu'il est colonisé par certains microorganismes vivants comme les bactéries, les cyanobactéries et les microalgues (SOLEIMANI et al., 2013 ; CHLAYON et al., 2020).

Sachant que la colonisation de matériau cimentaire par les microorganismes forme une étape primordiale de l'adhésion d'autres organismes, la finalité de la démarche est de favoriser la colonisation du support pour diminuer l'impact écologique de l'ouvrage et par voie de conséquences augmenter la biodiversité, sans impacter les exigences de résistance et de durabilité des ouvrages éco-conçus. Par conséquent, dans le but d'étudier la bioréceptivité du béton dans l'environnement marin, cet article se focalise successivement sur les caractéristiques du milieu marin qui influent sur sa colonisation et sur les microorganismes concernés. Ensuite, les caractéristiques intrinsèques du béton susceptibles d'influencer la bioréceptivité sont listées et décrites de même que les mécanismes conduisant à la formation du biofilm et à la biocolonisation des bétons. Enfin, le sujet de l'effet positif (bioprotection) ou négatif (biodéterioration) des organismes vivants sur le béton est abordé.

Tableau 1. Les principaux ouvrages en béton utilisés en site maritime.

\begin{tabular}{|c|c|c|}
\hline Types d'ouvrages & Objectifs fonctionnels & Matériaux \\
\hline \multicolumn{3}{|c|}{ Ouvrages de défense de côte et des ports } \\
\hline $\begin{array}{l}\text { Brise-lames } \\
\text { Epis } \\
\text { Digues, jetée }\end{array}$ & $\begin{array}{l}\text { Ouvrage de défense pour les } \\
\text { protéger des actions de la mer et } \\
\text { de l'érosion }\end{array}$ & $\begin{array}{l}\text { Enrochements } \\
\text { Blocs en béton }\end{array}$ \\
\hline \multicolumn{3}{|c|}{ Ouvrages portuaires pour service portuaire } \\
\hline $\begin{array}{l}\text { Appontement, } \\
\text { estacades, wharfs, } \\
\text { quais }\end{array}$ & $\begin{array}{l}\text { Ouvrage d'accostage permettant } \\
\text { le chargement et le } \\
\text { déchargement des navires }\end{array}$ & $\begin{array}{l}\text { Tablier en béton armé sur } \\
\text { piles }(B A) \text { ou sur pieux } \\
\text { métalliques } \\
\text { Ecrans de soutènement }\end{array}$ \\
\hline $\begin{array}{l}\text { Ponton } \\
\text { Ponton lourd brise- } \\
\text { clapot }\end{array}$ & $\begin{array}{l}\text { Structure flottante permettant } \\
\text { l'amarrage et l'accostage des } \\
\text { bateaux dans un port et } \\
\text { protection de l'agitation }\end{array}$ & $\begin{array}{l}\text { Métal ou béton armé, tenus } \\
\text { sur pieux métalliques ou } \\
\text { corps morts en béton armé }\end{array}$ \\
\hline \multicolumn{3}{|c|}{ Ouvrages en mer ouverte } \\
\hline Ancrages, lests & $\begin{array}{l}\text { Structures sous-marines pour } \\
\text { l'ancrage d'un navire ou d'une } \\
\text { infrastructure flottante }\end{array}$ & Béton armé \\
\hline Récifs artificiels & $\begin{array}{l}\text { Structures sous-marines } \\
\text { destinées à la protection ou la } \\
\text { production de faunes et de flore } \\
\text { ou de récréation }\end{array}$ & $\begin{array}{l}\text { Béton armé, métal, } \\
\text { matériaux divers }\end{array}$ \\
\hline
\end{tabular}




\section{Les facteurs biologiques de biocolonisation des bétons en milieu marin}

\subsection{La colonisation et le développement du biofilm à la surface du béton}

L'écosystème marin est considéré comme une unité de l'environnement dans laquelle une communauté biologique est entretenue grâce à la lumière du soleil, qui est nécessaire à la photosynthèse de certains organismes (SIGG et al., 2001). En plus de la luminosité, l'eau de mer contient des micro-nutriments et des macro-nutriments essentiels pour la croissance d'autres microorganismes vivants non photosynthétiques comme les bactéries. Les macro-nutriments qui sont constitués de carbone $(\mathrm{C})$, phosphore $(\mathrm{P})$, azote $(\mathrm{N})$, et silicium( $\mathrm{Si})$, fournissent les éléments nutritifs indispensables aux fonctions métaboliques des organismes vivants. Les micro-nutriments, comme le fer (Fe), le Zinc ( $\mathrm{Zn}$ ), le cuivre $(\mathrm{Cu})$ ou le manganèse $(\mathrm{Mn})$, bien qu'en plus faible quantité, jouent le rôle de co-facteurs enzymatiques pour les réactions métaboliques (PASQUERON DE FOMMERVAULT, 2016). Pour ces raisons, l'eau de mer est riche en macroorganismes mais aussi en microorganismes, tels que les bactéries, les cyanobactéries et les microalgues (POLI et al., 2017). À noter que la concentration et la diversité des organismes marins dépendent aussi d'autres conditions environnementales telles que la pression, la température et la salinité. La biodiversité est moins importante dans le cas où ces conditions sont plus drastiques et alors plus sélectives vis-à-vis des organismes (pression de sélection) (BOEUF, 2016).

Dès les premières heures qui suivent l'immersion d'un objet dans l'eau de mer, un matériau cimentaire est recouvert d'un biofilm composé d'une ou de plusieurs communautés microbiennes (DANG \& LOVELL, 2015).

Par définition, le biofilm est considéré comme étant une communauté microbienne sessile attachée d'une manière irréversible à un substrat ou une interface et incluse dans une gangue de substances polymériques extracellulaires, EPS (Extracellular Polymeric Substance). En effet, ces cellules présentent des phénotypes modifiés par rapport aux cellules planctoniques qui flottent librement dans le milieu (DONLAN \& COSTERTON, 2002).

Le biofilm constitue donc la première étape de colonisation qui facilite ensuite l'adhésion et l'installation des autres organismes marins notamment grâce aux EPS (WOOD et al., 2016). Les autres microorganismes (mycètes et microalgues), puis les macroorganismes (macroalgues et invertébrés) viennent s'implanter au fil du temps en complément ou en lieu et place du biofilm (SALTA et al., 2013 ; BRIAN-JAISSON et al., 2014). 


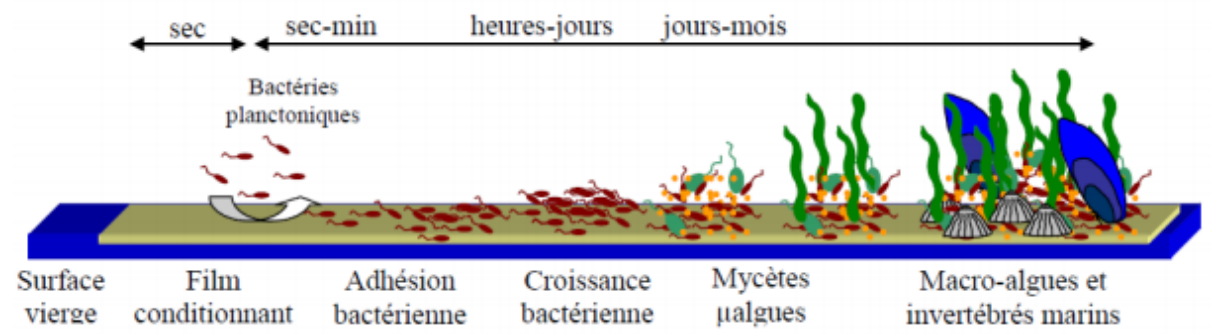

Figure 2. Biofouling en milieu marin (HARAS, 2005).

Toutefois, après immersion, une phase d'induction permet le conditionnement de la surface (formation $\mathrm{du}$ film conditionnant), suivi d'une phase d'accumulation exponentielle caractérisée par l'adhésion et la croissance des bactéries et d'autres microorganismes sur la surface (HARAS, 2005). Cette phase d'induction est similaire à la phase de latence observée classiquement en microbiologie lors de culture bactérienne au laboratoire. Elle est alors plus ou moins longue en fonction des paramètres environnementaux du milieu et des paramètres intrinsèques du matériau d'accueil (KARUNASAGAR et al., 1996 ; HAYEK et al., 2019). Ainsi, un support solide immergé est le siège de l'adsorption de molécules organiques ou inorganiques et d'ions, ce qui entraîne une modification des propriétés physico-chimiques de la surface, formant ainsi une micro-niche (sur une petite échelle par comparaison au matériau) riche en nutriments, favorable à l'adhésion des cellules bactériennes. Le conditionnement de la surface impacte alors l'adhésion des cellules bactériennes qui peut être stimulée ou inhibée par la nature des molécules adsorbées. C'est pourquoi cette étape est considérée comme une étape clé dans la formation du biofilm (TANG et al., 2006).

La formation de biofilm bactérien est donc un processus complexe, et à ce jour, il n'existe pas de modèle universel de la formation d'un biofilm. Toutefois les étapes clés sont (i) l'adhésion réversible et irréversible des cellules bactériennes à la surface du matériau cimentaire (ii) la croissance et la maturation du biofilm avec la sécrétion des EPS (iii) puis son détachement partiel et la dispersion des cellules bactériennes (O'TOOLE et al., 2000).

Par ailleurs, l'adhésion des bactéries sur une surface nécessite d'abord un déplacement de celles-ci vers la surface à coloniser. Ce déplacement peut être obtenu grâce à des phénomènes environnementaux (forces de gravité et forces hydrodynamiques du fluide environnant) et/ou par des appendices bactériens générateurs de mouvement, tels que les flagelles ou les pilis bactériens (HA \& O'TOOLE, 2015).

Une fois que l'organisme atteint une distance critique par rapport au support (généralement $<1 \mathrm{~nm}$ ), les bactéries établissent des interactions faibles avec cette surface (forces électrostatiques, forces de Van der Waals et interactions hydrophobes) (CARPENTIER \& CERF, 1993 ; DUNNE, 2002). Selon ces différentes interactions, la bactérie peut adhérer ou bien être emportée sous l'effet d'actions hydrodynamiques. Si 
ces interactions sont favorables, la bactérie adhère sur la surface et cette étape est considérée alors comme la phase d'adhésion réversible (HARAS, 2005). Les facteurs qui influencent cette phase sont ceux qui interagissent entre les cellules bactériennes et la surface du matériau cimentaire (i) d'une part les caractéristiques propres des bactéries (la charge de surface qui est généralement négative, l'hydrophobicité de sa surface, la composition chimique de la paroi cellulaire...) (ii) d'autre part celles de la surface d'accueil (nature physico-chimique, polarité, porosité, rugosité de surface...) (iii) et enfin les conditions environnementales ( $\mathrm{pH}$, température et conditions de flux) (SPERANZA et al., 2004). Selon CARPENTIER and CERF (1993), une surface chargée négativement pourrait avoir un effet répulsif, car la plupart des bactéries sont chargées négativement. En revanche, la porosité et la rugosité sont réputées comme augmentant l'adhésion bactérienne.

L'adhésion devient ensuite irréversible lorsque des interactions covalentes fortes sont établies entre la bactérie et la surface du matériau cimentaire, grâce aux macromolécules de surfaces bactériennes, telles que les polysaccharides, les protéines ou les lipopolysaccharides (HARAS, 2005).

Une fois en place sur le support, les bactéries se multiplient en synthétisant et développant la structure 3D de leur EPS pour former des colonies qui recouvrent tout ou partie de la surface. Cette étape varie selon les bactéries en présence et en fonction de (i) leur capacité à communiquer entre elles (système de communication de type quorum sensing) (DOBRETSOV et al., 2011) (ii) la concentration intracellulaire de c-di-GMP (une concentration intracellulaire élevée en c-di-GMP favorise l'expression des gènes impliqués dans la formation de biofilm) (DANG \& LOVELL, 2015) (iii) les conditions du milieu (iv) les propriétés du support notamment son caractère hydrophobe (O'TOOLE et al., 2000 ; HARAS, 2005).

À maturité, le biofilm a une structure tridimensionnelle entourée par une matrice composée des éléments minéraux et de substances polymériques extracellulaires (EPS) : protéines, polysaccharides, acides nucléiques, lipides et autres composants cellulaires. Cette matrice forme une barrière protectrice pour les microorganismes du biofilm contre les menaces extérieures (SOLEIMANI et al., 2013 ; HA and O'TOOLE, 2015).

La dernière étape du développement du biofilm est la dispersion de cellules dans l'environnement après le détachement des cellules du support. Cette dispersion est une partie essentielle du cycle du biofilm, car elle permet aux bactéries de coloniser de nouvelles surfaces, afin de redévelopper un nouveau biofilm (KAPLAN, 2010). En général, les mécanismes de dispersion du biofilm peuvent être divisés en deux grandes catégories (i) ceux correspondant à la dispersion active qui est basée sur des mécanismes initiés par les bactéries elles-mêmes (ii) ceux qui sont liés à la dispersion passive due à des forces extérieures, telles que les forces de cisaillement dues aux conditions hydrodynamiques (LAWRENCE et al., 2002). Plusieurs facteurs sont impliqués dans le processus complexe de dispersion du biofilm, tels que les signaux environnementaux, les 
systèmes de quorum sensing, et la production par les bactéries d'enzymes extracellulaires pouvant dégrader les composants de la matrice du biofilm facilitant ainsi sa dissociation (KAPLAN, 2010).

Généralement, le développement de biofilm sur une surface cimentaire est relativement long, car les bétons présentent un pH élevé de l'ordre de 13. Cette alcalinité hautement basique est défavorable aux attaques biologiques parce que peu de microorganismes peuvent se développer à ces $\mathrm{pH}$ très basiques. Pour cela, l'étape d'induction, préliminaire à la colonisation des matériaux cimentaires par les microorganismes et les macroorganismes doit s'établir, afin de diminuer le $\mathrm{pH}$ de 13 vers 9,5, $\mathrm{pH}$ à partir duquel la croissance de microorganismes devient possible (ROBERTS et al., 2002). Cette modification du $\mathrm{pH}$ de surface est due à l'adsorption des composés minéraux et organiques sur la surface ainsi qu'à la réaction du béton avec l'air (lors de sa fabrication et lors de son immersion) et l'eau de mer qui contient plusieurs espèces dissoutes dont la concentration est élevée. Le magnésium, le sulfate et les ions chlorures, ainsi que du dioxyde de carbone sont particulièrement connus pour attaquer la portlandite $\mathrm{Ca}(\mathrm{OH})_{2}$, le monosulfoaluminate de calcium hydraté et le silicate de calcium hydraté (C-S-H : "Calcium silicate hydrate") de liant hydraté (SANTHANAM \& OTIENO, 2016 ; HARSHADA et al., 2017). Le magnésium présent dans l'eau de mer sous forme de $\mathrm{MgSO}_{4}$ (sulfate de magnésium) et $\mathrm{MgCl}_{2}$ (chlorure de magnésium) attaque la portlandite et aboutit à la précipitation de brucite insoluble $\mathrm{Mg}(\mathrm{OH})_{2}$ et à la lixiviation (une substitution entre l'ion $\mathrm{Ca}^{2+}$ et $\mathrm{Mg}^{2+}$ ) des C-S-H qui deviennent progressivement des M$\mathrm{S}-\mathrm{H}$, (Magnésium silicate hydrate) moins compacts et résistants (LORS et al., 2017). Le sulfate sous sa forme $\left(\mathrm{CaSO}_{4}\right)$ réagit avec les aluminates du liant et conduit alors à la précipitation d'ettringite expansive. Le dioxyde de carbone présent dans l'eau sous forme de bicarbonate de potassium se combine à la portlandite en produisant des carbonates de calcium.

DE WEERDT et al., (2014) ont montré qu'en une vingtaine de jours, le liant cimentaire immergé dans l'eau de mer subit des changements élémentaires de phases indépendamment du type de liant testé.

\subsection{Les microorganismes colonisateurs des matériaux cimentaires}

En environnement marin, les microorganismes colonisateurs sont nombreux, ubiquistes et susceptibles de former un biofilm sur les surfaces des matériaux dans des conditions convenables. Les principaux microorganismes colonisateurs des matériaux cimentaires sont les bactéries, les champignons, les cyanobactéries et les microalgues (GAYLARDE \& GAYLARDE, 2005).

\subsubsection{Les bactéries}

Les bactéries sont des microorganismes unicellulaires de taille moyenne, variant de 0,5 à 1,5 $\mu \mathrm{m}$ (CANLER et al., 2011), qui se développent sur tout type de support et sont 
généralement pionnières dans le processus de biocolonisation (HARAS, 2005). Le biofilm qu'elles constituent représente généralement le point de départ de la biocolonisation et des différentes interactions matériaux - microorganismes pouvant avoir lieu (HARAS, 2005 ; HA \& O'TOOLE, 2015).

Les phénomènes de détérioration des matériaux cimentaires par induction microbiologique sont connus depuis l'année 1945 suite aux travaux de PARKER (1945) qui a été le premier à isoler à partir d'échantillons de béton une espèce bactérienne productrice d'acide sulfurique appelée initialement Thiobacillus concretivorus et désormais Acidithiobacillus thiooxidans. Ce phénomène est majoritairement étudié dans les réseaux d'assainissement où le milieu riche en sulfates et en nutriments rend les conditions optimales au développement d'une faune microbienne appartenant au genre Thiobacillus (YUAN et al., 2015). Les Thiobacilli sont des bactéries sulfo-oxydantes qui oxydent les composés soufrés en acide sulfurique, substance particulièrement agressive pour les bétons (DE BELIE, 2010).

Les processus biochimiques essentiels amenant à la dégradation des bétons sous l'influence des bactéries reposent essentiellement sur la synthèse d'acides tels que l'acide sulfurique $\left(\mathrm{H}_{2} \mathrm{SO}_{4}\right)$, le sulfure d'hydrogène et l'acide nitrique $\mathrm{HNO}_{3}$ (CWALINA, 2008). Les acides tels que l'acide sulfurique et l'acide nitrique dissolvent les éléments calcaires ce qui a pour conséquence une perte de cohésion du matériau, l'apparition de fissures ou encore la corrosion des armatures présentes dans le béton (LORS et al., 2017). Ces effets sont usuels dans les réseaux d'assainissement où le milieu est riche en sulfate et ammoniac.

Malgré le nombre important d'ouvrages en béton présents dans l'eau de mer, l'eau douce des rivières et l'eau des lacs, les études concernant la colonisation de matériaux cimentaires par les bactéries de ces environnements restent peu nombreuses (SOSA et al., 2011).

\subsubsection{Les microalgues et les cyanobactéries}

Les algues microscopiques (micro-algues) et les cyanobactéries désignent une série d'espèces unicellulaires ou pluricellulaires, de taille généralement comprise entre $0,5 \mu \mathrm{m}$ et $1 \mathrm{~mm}$. Ces microorganismes sont caractérisés par leur capacité à réaliser la photosynthèse oxygénique (la photosynthèse est la réaction qui permet à certains organismes vivants de synthétiser de la matière organique à partir de $\mathrm{CO}_{2}$, d'un donneur d'électrons en présence de lumière). Les algues et les cyanobactéries sont donc des organismes autotrophes, qui sont capables de générer leurs propres matières organiques à partir d'éléments minéraux (CALLEN \& PERASSO, 2005).

Selon la littérature, les algues vertes (Chlorophycées), les cyanobactéries (Cyanophytes) sont responsables des salissures du béton de couleurs verdâtres, rougeâtres ou noirâtres selon la composition du biofilm. Par ailleurs, la sécrétion d'acides organiques résultant du métabolisme de ces algues et des cyanobactéries pourrait conduire à la dissolution du 
carbonate de calcium des pierres calcaires, bétons et mortiers. Les algues et cyanobactéries peuvent être responsables de bioaltération et de biodégradation (ESCADEILLAS et al., 2007).

Les cyanobactéries sont des microorganismes procaryotes alors que les microalgues sont des microorganismes eucaryotes. Celles-ci sont réparties entre plusieurs groupes phylogénétiques différents, tels que les chlorophytes, les rhodophytes et les diatomées (Bacillariophyta) (DEJOYE-TANZI, 2013). Les chlorophytes se développent majoritairement dans les eaux douces, telles que les rivières et représentent le groupe principal d'algues vertes (PENCREAC'H et al., 2004) tandis que les rhodophytes sont principalement des organismes marins pluricellulaires de dimensions macroscopiques appelées algues rouges (HAMIDOUCHE et al., 2017). Les diatomées sont des organismes unicellulaires de taille microscopique caractérisés par un squelette siliceux appelé "frustule" qui les enveloppe. Elles sont abondantes en milieux marins (ROBERT et al., 2004).

La biocolonisation des matériaux cimentaires in situ par des cyanobactéries et des algues a été confirmée par la mesure de la quantité de chlorophylle et/ou par l'apparition des taches de couleurs différentes sur les échantillons testés dans diverses études (SILVA and NAIK, 2013 ; VISHWAKARMA et al., 2014). Dans la plupart de ces études, les espèces colonisatrices n'ont pas été identifiées et caractérisées, d'où la nécessité d'avoir plus d'études et d'investissement amenant à une compréhension plus profonde de l'interaction entre les matériaux cimentaires et les microorganismes dans le milieu marin.

\subsubsection{Les champignons}

Les champignons, dont la taille varie de 1 à $100 \mu \mathrm{m}$, sont eucaryotes chimiotrophes. Ainsi, ils ne sont pas capables de réaliser la photosynthèse pour fabriquer leurs propres matières organiques. Pour se développer, les champignons nécessitent des milieux humides, et leur énergie provient des réactions d'oxydo-reductions. Ils sont peu sensibles à la variation de $\mathrm{pH}$, mais se développent plus facilement à $\mathrm{pH}$ acide.

Les champignons sont hétérotrophes. En effet, ils ne peuvent synthétiser leurs constituants cellulaires qu'à partir d'une source de carbone organique. Une colonisation fongique sur les matériaux cimentaires nécessite donc une accumulation de matière organique à la surface. Dans le cas d'une colonisation réussie, les champignons peuvent conduire à la bioaltération ou à la biodéterioration du matériau colonisé. Sous l'effet de leur développement et de leur métabolisme, certains champignons produisent des pigments (par exemple, des mélanines), qui provoquent l'apparition des taches foncées amenant ainsi à la bioaltération du matériau cimentaire colonisé (GIANNANTONIO et al., 2009). De plus, les champignons produisent une grande quantité d'acides organiques (acide acétique, formique, oxalique et tartrique), qui sont responsables de la solubilisation du calcaire dans les matrices minérales et de la chélation des ions calcium, mais aussi d'ions métalliques, tels que l'aluminium amenant à la détérioration du matériau. 
Le milieu marin est considéré comme un milieu contenant un nombre important d'organismes fongiques (SANTHANAM \& OTIENO, 2016). Cependant les études concernant la colonisation des matériaux cimentaires dans l'environnement marin par des champignons restent très peu nombreuses. Les surfaces immergées sont souvent couvertes par une ou plusieurs couches d'algues et d'autres organismes protozoaires (DÜRR \& THOMASON, 2009), ce qui conduit à rendre difficile l'identification et l'isolement des champignons sessiles (colonisateurs) en milieu marin sans l'utilisation des techniques de biologie moléculaire (extraction et séquençage des ARN 18S) qui nécessitent encore plus d'investissement.

\section{Les facteurs physico-chimiques du matériau influençant la biocolonisation de la surface cimentaire dans le milieu marin}

La colonisation et le développement de biofilm sur une surface cimentaire ont fait l'objet de plusieurs études montrant que la biocolonisation d'une surface est induite et gouvernée par l'interaction de trois acteurs: le matériau, les microorganismes vivants et l'environnement dans lequel se trouvent les deux premiers (LORS et al., 2017).

Pour cela, deux types de bioréceptivité sont définis : la bioréceptivité intrinsèque, liée uniquement aux propriétés physico-chimiques de la surface du béton (porosité, rugosité...) et à la composition chimique du matériau, et la bioréceptivité extrinsèque relative à l'environnement (LORS et al., 2017).

De nombreux travaux montrent que la nature du matériau affecte la cinétique de biocolonisation, la distribution des cellules bactériennes adhérentes et la formation du biofilm. Les différences observées entre matériaux peuvent être induites par la nature chimique du matériau, mais surtout par leurs propriétés physico-chimiques ou leur topographie (BELLON-FONTAINE et al., 2016). Les propriétés physico-chimiques d'un matériau cimentaire vont donc influer sur la bioréceptivité d'une manière directe ou indirecte.

\subsection{La composition chimique}

Selon sa composition chimique, un matériau peut contenir des substances favorisant ou inhibant le développement des microorganismes. Par exemple, la présence de certains métaux lourds, en quantité suffisante, peut inhiber la croissance et le développement des microorganismes (DUBOSC et al., 2001).

Néanmoins, SOUCHE et al., (2019) ont réalisé des tests de lixiviation sur bétons pour vérifier la capacité de relargage de substances toxiques dans de l'eau déminéralisée et dans de l'eau de mer. Dans l'eau de mer, aucun relargage n'a été constaté après immersion des échantillons de bétons. En outre, les paramètres de formulation d'un béton peuvent influencer des paramètres tels que la rugosité, la porosité ou encore le pH (LORS et al., 2017 ; PERKOL-FINKEL \& SELLA 2014). 


\subsection{La rugosité de la surface}

La rugosité de surface des matériaux est l'un de principaux facteurs physiques influençant sa bioréceptivité. La rugosité se traduit par des aspérités et des irrégularités en surface du matériau (PIOCH et al., 2018).

Ces irrégularités peuvent former des sites d'ancrage et des micro-refuges pour l'installation des microorganismes, ce qui favorise ainsi la biocolonisation (AMMAR et al., 2015). Ces micro-refuges protègent les microorganismes contre les forces hydrodynamiques ayant tendance à les arracher surtout lors de l'adhésion réversible, très fragile (BELLON-FONTAINE et al., 2016). KERR et al., (1999) ont observé une augmentation de l'adhésion bactérienne sur des échantillons de verre et d'acrylique immergés en eau de mer naturelle lorsque la rugosité augmente de 0,25 à $30 \mu \mathrm{m}$. TEUGHELS et al., (2006) ont montré que la formation de plaque dentaire est favorisée sur une surface rugueuse. Concernant les matériaux cimentaires, plusieurs études ont démontré l'effet de la rugosité de surface sur l'encrassement biologique (TRAN et al., 2014 ; MANSO \& AGUADO, 2016 ; SOUCHE et al., 2019). Ces études ont montré que les surfaces cimentaires rugueuses ont une bioréceptivité plus importante que les surfaces lisses dans des conditions contrôlées de laboratoire ou dans des conditions naturelles.

Outre son effet sur l'adhésion des microorganismes, la rugosité de surface influe également sur la vitesse d'écoulement de l'eau ainsi que sur l'adsorption des matières organiques (DUBOSC et al., 2001 ; EL-NAGGAR et al., 2010). Cela impacte directement la formation du film conditionnant pendant la phase d'induction. Certaines études nuancent l'effet de la rugosité sur la bioréceptivité du matériau en précisant que cet effet dépend des caractéristiques des microorganismes en question (types de microorganisme, sa géométrie, sa taille, sa forme...) (WHITMAN et al., 1998). Par exemple, les microalgues filamenteuses, comme Klebsormidium flaccidum, s'accrochent aux surfaces rugueuses tandis que les microalgues à parois épaisses adhèrent aux surfaces lisses (TRAN et al., 2014 ; LORS et al., 2017).

\subsection{La porosité totale à l'eau}

Dans le cas de supports non immergés dans l'eau, les bactéries colonisent préférentiellement les supports poreux. Ce constat s'explique principalement par l'influence de la porosité sur l'absorption et la rétention de l'eau par le matériau déterminant pour le développement des microorganismes (NUHOGLU et al., 2006). Des infractuosités de la taille du microorganisme concerné semblent idéales. Plus petites elles aident à l'ancrage, mais ne protègent pas des flux. Trop grandes elles ne permettent pas un transfert optimum des nutriments.

Dans le cas de bétons immergés, il est difficile de déterminer l'effet de la porosité sur la bioréceptivité (TRAN et al., 2014). Cette difficulté a été expliquée par la saturation permanente des échantillons testés en eau et donc par une humidité élevée tout au long de l'immersion. 


\subsection{Le pH de la surface}

Le $\mathrm{pH}$ de la surface du matériau peut définir la nature et la capacité d'un microorganisme à se fixer sur celle-ci (SHI \& ZHU, 2009). Généralement, chaque espèce a un pH optimal de croissance et peut se développer dans une gamme définie plus ou moins étendue autour de ce $\mathrm{pH}$. Selon ces gammes, les microorganismes sont classés en : microorganismes acidophiles $(\mathrm{pH}<6)$, neutrophiles $(6<\mathrm{pH}<8)$ ou basophiles $(\mathrm{pH}>8)$.

Les activités métaboliques des microorganismes, leur croissance, leur adhésion et la synthèse d'EPS sont influencées par le $\mathrm{pH}$ à la surface du matériau. La réduction du $\mathrm{pH}$ de surface d'un béton après immersion dans l'eau de mer qui possède un $\mathrm{pH}$ légèrement basique de l'ordre de 8 (de 13 vers environ 9) accélère la biocolonisation de la surface et améliore la bioréceptivité du matériau (ROBERTS et al., 2002 ; TRAN et al., 2014).

\subsection{L'hydrophobicité de surface}

Les microorganismes hydrophiles se fixent plus facilement à des surfaces hydrophiles tandis que les microorganismes hydrophobes préfèrent des surfaces hydrophobes (SHI \& ZHU, 2009). À noter que l'hydrophobicité et la charge de la surface dépendent de la composition chimique des matériaux. Selon plusieurs études, cette composition influe sur le type et la diversité des microorganismes colonisateurs (GIANNANTONIO et al., 2009 ; MILLER et al., 2012).

Malgré l'importance de ce paramètre sur la biodiversité marine ainsi que sur la biocolonisation de support immergé, les études qui mettent en relation l'hydrophobicité de surface des matériaux cimentaires et la biocolonisation dans le milieu marin semblent être inexistantes à ce jour.

\section{La biocolonisation des bétons dans le milieu marin, un effet positif sur la durabilité ?}

Dès que l'infrastructure maritime est conçue pour devenir un habitat favorable à l'installation d'organismes vivants, il faut évaluer les interactions entre la biocolonisation et la durabilité des ouvrages. En effet, l'installation de microorganismes et de macroorganismes modifie la zone d'interface entre le matériau et son milieu. Cette biocolonisation peut être à l'origine d'un effet positif ou négatif comme la bioprotection ou la biodégradation du matériau (HARSHADA et al., 2017).

Bien que la biocolonisation des ouvrages maritimes en béton soit spontanée, relativement peu d'attention a été accordée à la biodégradation de béton dans le milieu marin. Cela peut être expliqué par les difficultés et les efforts nécessaires pour séparer la biodétérioration d'autres processus de dommages de matériaux cimentaires dans l'eau de mer (vieillissement, attaques chimiques par les chlorures ou par les sulfates...etc.). FERRERO a démontré en 2018 par des expériences effectuées au laboratoire que la biocolonisation de matériaux cimentaires par des microorganismes comme la diatomée 
Cylindrotheca closteriumque permet une pénétration plus importante des ions chlorure et sulfate dans le matériau cimentaire, ce qui accélère la dégradation du matériau. Selon FERRERO (2018), la création d'un micro-réseau dans le matériau par les diatomées pourrait expliquer ce phénomène.

Sachant que le milieu marin est extrêmement diversifié, les résultats des expériences effectués au laboratoire ne reflètent pas toujours la réalité. L'effet de la biocolonisation observé en laboratoire est fortement dépendant du choix de microorganismes, du matériau d'étude (béton, mortier, pâte de ciment, ciment Portland, ciment laitier...) ainsi que les conditions expérimentales établies. Selon LV et al., (2015), la biocolonisation du béton peut améliorer sa durée de vie en fonction du substrat et des microorganismes impliqués. Ces auteurs montrent que le biofilm formé par des bactéries du genre Pseudoalteromonas sur la surface du matériau cimentaire améliore sa durabilité par la réduction de la quantité d'ions agressifs $\left(\mathrm{Cl}^{-}, \mathrm{Mg}^{2+}, \mathrm{OH}^{-}\right)$pouvant pénétrer dans le matériau et induire sa biodétérioration. Cet effet de bioprotection a été identifié non seulement dans le cas de biofilm bactérien au laboratoire, mais aussi in situ dans le cas de biofilm constitué par d'autres microorganismes et macroorganismes marins, tels que les cyanobactéries, les algues, les lichens et les balanes (BERTRON, 2014). Ces organismes vivants réduisent ainsi la perméabilité du matériau cimentaire, ce qui conduit à une meilleure durabilité du matériau cimentaire (COOMBES et al., 2017). La biocolonisation pourrait alors devenir une "bioprotection" des structures en fournissant à l'ouvrage ou au matériau une protection supplémentaire contre les agressions chimiques, voire mécaniques, grâce aux effets de la barrière physique offerte par les organismes vivants, qui colonisent la surface du matériau cimentaire (CHLAYON et al., 2020). Selon la littérature, un biofilm bénéfique peut protéger le matériau par la réduction de la quantité d'eau et de nutriments disponibles, par la synthèse des agents anti-microbiens (ce qui inhibe la croissance d'autres microorganismes et macroorganismes ayant un effet néfaste sur les matériaux cimentaires) ou par la formation d'une couche protectrice via les exopolysaccharides synthétisés (SOLEIMANI et al., 2013).

De plus, le biofilm microbien facilite l'installation d'autres organismes vivants sur la surface. Dans le cas de matériaux cimentaires, l'attachement des organismes vivants comme les huîtres, les vers serpulides, les balanes et les coraux peuvent induire la formation de calcite précipitant dans les vides de la matrice cimentaire et rendant le béton plus dense et plus résistant (JOSHI et al., 2018).

La biocolonisation de la surface d'un béton pourrait donc réduire leur détérioration suite aux agressions environnementales (COOMBES et al., 2013 ; COOMBES et al., 2017). De plus, la biocolonisation des bétons amène une stabilisation thermique au matériau cimentaire (COOMBES et al., 2013). Ainsi, cette bioprotection semble améliorer la durabilité des matériaux de construction en protégeant la surface du béton de l'érosion mécanique des vagues et de la houle ainsi que des agressions physico-chimiques (rayonnement UV solaire, sels minéraux...). 


\section{Conclusions et perspectives}

En accord avec la démarche d'écoconception des ouvrages à la mer, l'étude des phénomènes de biocolonisation du béton dans l'eau de mer doit se poursuivre dans un double but, la durabilité du matériau et l'impact sur l'environnement. Il s'agit de maîtriser les processus biologiques de développement des microorganismes marins (bactéries, champignons et microalgues) et les caractéristiques physico-chimiques du matériau (rugosité, $\mathrm{pH}$, composition chimique, porosité, hydrophobicité...) dans l'environnement local d'un ouvrage.

Les effets positifs pour la nature pourraient se cumuler avec les effets positifs pour la durabilité des ouvrages, en offrant une bioprotection dans certaines conditions. La maîtrise des facteurs influençant la biocolonisation voir plus tard la durabilité des matériaux cimentaires dans l'environnement marin doit pouvoir influer sur le positionnement des donneurs d'ordres, sur les méthodologies d'ingénierie maritime en usage à ce jour et proposer des pistes intéressantes d'amélioration dans la conception et la mise en œuvre de bétons à vocation maritime, en particulier dans le contexte de l'approche performantielle des bétons.

\section{Références bibliographiques}

AMMAR Y., SWAILES D., BRIDGENS B., CHEN J. (2015). Influence of surface roughness on the initial formation of biofilm. Surface and Coatings Technology, Vol. 284, pp 410-416. https://dx.doi.org/10.1016/j.surfcoat.2015.07.062

BELLON-FONTAINE M.N., BENEZECH T., BOUTROUX K., HERMON C. (2016). Conception hygiénique de matériel et nettoyage-désinfection, Coll. Sciences et techniques agroalimentaires. Lavoisier collection Tec \& Doc, 224 p.

BERTRON A. (2014). Understanding interactions between cementitious materials and microorganisms: a key to sustainable and safe concrete structures in various contexts. Materials and Structures, Vol. 47, pp 1787-1806. http://dx.doi.org/10.1617/s11527-014-0462-9 BOEUF G. (2016). La biodiversité dans l'océan. Planet-Vie.

https://planet vie.ens.fr/thematiques/themes-transversaux/la-biodiversite-dans-l-ocean BRIAN-JAISSON F., ORTALO-MAGNE A., GUENTAS-DOMBROWSKY L., ARMOUGOM F., BLACHE Y., MOLMERET M. (2014). Identification of bacterial strains isolated from the Mediterranean sea exhibiting different abilities of biofilm formation. Microbial Ecology, Vol. 68, pp 94-110. https://doi.org/10.1007/s00248-013-0342-9 CALLEN J.-C., PERASSO R. (2005). Biologie cellulaire - Des molécules aux organismes. Collection Sciences de la vie, Dunod, 2ème édition, 512 p.

CANLER J.P., PERRET J.-M., DUCHENE P. (2011). Aide au diagnostic des stations d'épuration par l'observation microscopique des: boues activées. Editions Quae, 160 p. 
CARPENTIER B., CERF O. (1993). Biofilms and their consequences, with particular reference to hygiene in the food industry. Journal of Applied Bacteriology, Vol. 75, pp 499-511. https://doi.org/10.1111/j.1365-2672.1993.tb01587.x

CHLAYON T., IWANAMI M., CHIJIWA N. (2020). Impacts from concrete microstructure and surface on the settlement of sessile organisms affecting chloride attack. Construction and Building Materials, Vol. 239, pp 117863. https://doi.org/10.1016/j.conbuildmat.2019.117863

COOMBES M.A., NAYLOR L.A., VILES H.A., THOMPSON, R.C. (2013). Bioprotection and disturbance: Seaweed, microclimatic stability and conditions for mechanical weathering in the intertidal zone. Geomorphology, Vol. 202, pp 4-14. https://doi.org/10.1016/j.geomorph.2012.09.014

COOMBES M.A., VILES H.A., NAYLOR L.A., LA MARCA E.C. (2017). Cool barnacles: Do common biogenic structures enhance or retard rates of deterioration of intertidal rocks and concrete? Science Total Environment, Vol. 580, pp 1034-1045. https://doi.org/10.1016/j.scitotenv.2016.12.058

CWALINA B. (2008). Biodeterioration of concrete. Architecture civil engineering environment, Vol. 4, pp 133-140.

DANG H., LOVELL C.R. (2015). Microbial surface colonization and biofilm development in marine environments. Microbiology and Molecular Biology Reviews Vol. 80, pp 91-138. https://doi.org/10.1128/MMBR.00037-15

DE BELIE N. (2010). Microorganisms versus stony materials: a love-hate relationship. Materials and Structures, Vol. 43, pp 1191-1202. https://doi.org/10.1617/s11527-010-9654-0

DEJOYE-TANZI C. (2013). Eco-extraction et analyse de lipides de micro-algues pour la production d'algo-carburant. Thèse Université d'Avignon et des Pays de Vaucluse, $177 \mathrm{p}$.

DE WEERDT K., JUSTNES H., GEIKER M.R. (2014). Changes in the phase assemblage of concrete exposed to sea water. Cement and Concrete Composites, Vol. 47, pp 53-63. https://doi.org/10.1016/j.cemconcomp.2013.09.015

DOBRETSOV S., TEPLITSKI M., BAYER M., GUNASEKERA S., PROKSCH P., PAUL V.J. (2011). Inhibition of marine biofouling by bacterial quorum sensing inhibitors. Biofouling, Vol. 27, pp 893-905. https://doi.org/10.1080/08927014.2011.609616

DONLAN R.M., COSTERTON J.W. (2002). Biofilms: Survival Mechanisms of Clinically Relevant Microorganisms. Clinical Microbiology Reviews, Vol. 15, pp 167193. https://doi.org/10.1128/CMR.15.2.167-193.2002

DUBOSC A., ESCADEILLAS G., BLANC P.J. (2001). Characterization of biological stains on external concrete walls and influence of concrete as underlying material. Cement and Concrete Research, Vol. 31, pp 1613-1617. https://doi.org/10.1016/S00088846(01)00613-5

DUNNE W.M. (2002). Bacterial Adhesion: Seen Any Good Biofilms Lately? Clinical Microbiology Reviews, Vol. 15, pp 155-166. https://doi.org/10.1128/CMR.15.2.155-166.2002 
DÜRR S., THOMASON J.C. (2009). Biofouling. Wiley on line edition, 429 p. https://doi.org/10.1002/9781444315462

EL-NAGGAR M.Y., WANGER G., LEUNG K.M., YUZVINSKY T.D., SOUTHAM G., YANG J., LAU W.M, NEALSON K.H., GORBY Y.A. (2010). Electrical transport along bacterial nanowires from Shewanella oneidensis MR-1. Proceedings of the National Academy of Sciences, Vol. 107, pp 18127-18131. https://doi.org/10.1073/pnas.1004880107 ESCADEILlAS G., BERTRON A., BLANC P., DUBOSC, A. (2007). Accelerated testing of biological stain growth on external concrete walls. Part 1: Development of the growth tests. Materials and Structures, Vol. 40, pp 1061-1071. https://doi.org/10.1617/s11527006-9205-x

FERRERO M.A. (2018). Colonisation et biodétérioration des bétons en milieu marin: mise au point d'essais en laboratoire et influence de la composition chimique du matériau cimentaire. Thèse Université de Caen Normandie, $223 \mathrm{p}$.

GAYLARDE C.C., GAYLARDE P.M. (2005). A comparative study of the major microbial biomass of biofilms on exteriors of buildings in Europe and Latin America. International Biodeterioration \& Biodegradation, Vol. 55, pp 131-139. https://doi.org/10.1016/j.ibiod.2004.10.001

GIANNANTONIO D.J., KURTH J.C., KURTIS K.E., SOBECKY P.A. (2009). Effects of concrete properties and nutrients on fungal colonization and fouling. International Biodeterioration \& Biodegradation, Vol. 63, pp 252-259. https://doi.org/10.1016/j.ibiod.2008.10.002

HA D.G., O'TOOLE G.A. (2015). c-di-GMP and its Effects on Biofilm Formation and Dispersion: a Pseudomonas Aeruginosa Review. Microbiology Spectrum, Vol. 3, pp 1-19. https://doi.org/10.1128/microbiolspec.MB-0003-2014

HAMIDOUCHE N., TETAH S. (2017). Contribution à l'étude de la flore algale en particulier les cyanobactéries de la lagune Tamelaht et du lac Mézaia. Rapport de Master, Université de Béjaia, $68 \mathrm{p}$.

HARAS D. (2005). Biofilms et altérations des matériaux: de l'analyse du phénomène aux stratégies de prévention. Matériaux \& Techniques, Vol. 93, pp s.27-s.41. https://doi.org/10.1051/mattech:2006003

HARSHADA M.K., DESAI P.M.M., GUPTA D.A.K. (2017). Deterioration of concrete in marine structure. Imperial Journal of Interdisciplinary Research (IJIR), Vol. 3(8), pp 198-209.

HAYEK M., BARAQUET C., LAMI R., BLACHE Y., MOLMERET M. (2019). The marine bacterium shewanella woodyi produces C8-HSL to regulate bioluminescence. Microbial Ecology, Vol. 79, pp 865-881. https://doi.org/10.1007/s00248-019-01454-z JOSHI S., GOYAL S., REDDY M.S. (2018). Influence of nutrient components of media on structural properties of concrete during biocementation. Construction and Building Materials, Vol. 158, pp 601-613. https://doi.org/10.1016/j.conbuildmat.2017.10.055 
KAPLAN J.B. (2010). Biofilm dispersal: mechanisms, clinical implications, and potential therapeutic uses. Journal of Dental Research, Vol. 89, pp 205-218. https://doi.org/10.1177/0022034509359403

KARUNASAGAR I., OTTA S.K., KARUNASAGAR I. (1996). Biofilm formation by Vibrio harveyi on surfaces. Vol. 140, pp 241-245. https://doi.org/10.1016/0044-8486(95)01180-3

KERR A., BEVERIDGE C.M., COWLING M.J., HODGKIESS T., PARR A.C.S., SMITH M.J. (1999). Some physical factors affecting the accumulation of biofouling. Journal of the Marine Biological Association of the United Kingdom, Vol. 79, pp 357359. https://doi.org/10.1017/S002531549800040X

LAWRENCE J.R., SCHARF B., PACKROFF G., NEU T.R. (2002). Microscale evaluation of the effects of grazing by invertebrates with contrasting feeding modes on river biofilm architecture and composition. Microbial Ecology, Vol. 44, pp 199-207. https://doi.org/10.1007/s00248-001-1064-y

LORS C., FEUGEAS F., TRIBOLLET B. (2017). Interactions MatériauxMicroorganismes: Bétons et métaux plus résistants à la biodétérioration. EDP Sciences LV J., MAO J., BA H. (2015). Influence of marine microorganisms on the permeability and microstructure of mortar. Construction and Building Materials, Vol. 77, pp 33-40. https://doi.org/10.1016/j.conbuildmat.2014.11.072

MANSO S., AGUADO A. (2016). The use of bio-receptive concrete as a new typology of living wall systems. Matériaux \& Techniques, Vol. 104, n5, article number 502, 8 p. https://oi.org/10.1051/mattech/2016028

MILLER A.Z., SANMARTÍN P., PEREIRA-PARDO L., DIONÍSIO A., SAIZJIMENEZ C., MACEDO M.F., PRIETO B. (2012). Bioreceptivity of building stones: A review. Science of The Total Environment, Vol. 426, pp 1-12. https://doi.org/10.1016/j.scitotenv.2012.03.026

NGUYEN V.H. (2005). Couplage dégradation chimique - comportement en compression du béton. Thèse Ecole des Ponts ParisTech, Paris, $220 \mathrm{p}$.

NUHOGLU Y., OGUZ E., USLU H., OZBEK A., IPEKOGLU B., OCAK I., HASENEKOGLU I. (2006). The accelerating effects of the microorganisms on biodeterioration of stone monuments under air pollution and continental-cold climatic conditions in Erzurum, Turkey. Science of The Total Environment, Vol. 364, pp 272283. https://doi.org/10.1016/j.scitotenv.2005.06.034

O'TOOLE G., KAPLAN H.B., KOLTER R. (2000). Biofilm formation as microbial development. Annual Review of Microbiology, Vol. 54, pp 49-79. https://doi.org/10.1146/annurev.micro.54.1.49

PARKER C.D. (1945). The corrosion of concrete: The isolation of a species of bacterium associated with the corrosion of concrete exposed to atmospheres containing hydrogen sulphide. Australian Journal of Experimental Biology and Medical Science, Vol. 23, pp 81-90. https://doi.org/10.1038/icb.1945.13 
PASQUERON DE FOMMERVAULT O. (2016). Dynamique des nutriments en Méditerranée: des campagnes océanographiques aux flotteurs Bio-Argo. Thèse Université Pierre et Marie Curie - Paris VI, Paris, 196 p.

PENCREAC'H G., DEVOS M., POISSON L., HERAULT J., LOISEAU C., ERGAN F. (2004). Les microalgues marines: source alternative d'acide eicosapentaènö̈que (EPA) et d'acide docosahexaènö̈que (DHA). Oléagineux, Corps gras, Lipides, Vol. 11, pp 118122. https://doi.org/10.1051/ocl.2004.0118

PERKOL-FINKEL S., SELLA I. (2014). Ecologically active concrete for coastal and marine infrastructure: innovative matrices and designs. From Sea to Shore - Meeting the Challenges of the Sea, ICE publishing, pp 1139-1149.

PIOCH S., RELINI G., SOUCHE J. C., STIVE M. J. F., DE MONBRISON D., NASSIF S., SIMARD F., ALLEMAND D., SAUSSOL P., SPIELER R., KILFOYLE K. (2018). Enhancing eco-engineering of coastal infrastructure with eco-design: Moving from mitigation to integration, Ecological Engineering. Vol 120, pp 574-584. https://doi.org/10.1016/j.ecoleng.2018.05.034

PLANETOSCOPE (2020). Panetoscope - Statistiques : Production mondiale de béton. Consultation du 28 février 2020. https://www.planetoscope.com/matierespremieres/1374-production-mondiale-de-beton.html

POLI A., FINORE I., ROMANO I., GIOIELLO A., LAMA L., NICOLAUS B. (2017). Microbial diversity in extreme marine habitats and their biomolecules. Microorganisms. Vol. 5, pp 2-25. https://doi.org/10.3390/microorganisms5020025

ROBERT R., CHRETIENNOT-DINET M. - J., KAAS R., MARTIN-JEZEQUEL V., MOAL J., LE COZ J.-R., NICOLAS J.-L., BERNARD E., CONNAN J.-P., LE DEAN L., LE GOURRIEREC G., LEROY B., QUERE C. (2004). Amélioration des productions phytoplanctoniques en écloserie de mollusques : caractérisation des microalgues fourrage. Rapport IFREMER, DRV/RA/RST/LPI/2004-05, 144 p.

ROBERTS D.J., NICA D., ZUO G., DAVIS J.L. (2002). Quantifying microbially induced deterioration of concrete: initial studies. International Biodeterioration \& Biodegradation, Vol. 49, pp 227-234. https://doi.org/10.1016/S0964-8305(02)00049-5

SALTA M., WHARTON J.A., BLACHE Y., STOKES K.R., BRIAND J.F. (2013). Marine biofilms on artificial surfaces: structure and dynamics. Environmental Microbiology, Vol. 15, pp 2879-2893. https://doi.org/10.1111/1462-2920.12186

SANTHANAM M., OTIENO M. (2016). Deterioration of concrete in the marine environment. Marine Concrete Structures, pp 137-149. https://doi.org/10.1016/B978-0-08-100081$\underline{6.00005-2}$

SHI X., ZHU X. (2009). Biofilm formation and food safety in food industries. Trends in Food Science \& Technology, Vol. 20, pp 407-413. https://doi.org/10.1016/j.tifs.2009.01.054 SIGG L., BEHRA P., STUMM W. (2001). Chimie des milieux aquatiques. Dunod édition, cinquième édition, $576 \mathrm{p}$. 
SILVA M.R., NAIK T.R. (2013). Biodeterioration of concrete structures in coastal zone. Third International Conference on Sustainable Construction Materials and Technologies, August 18 - August 21 2013, Kyoto Research Park, Kyoto, Japan Materials Research, Vol. 12,8 p.

SOLEIMANI S., ORMECI B., ISGOR O.B. (2013). Growth and characterization of Escherichia coli DH5a biofilm on concrete surfaces as a protective layer against microbiologically influenced concrete deterioration (MICD). Applied microbiology and biotechnology, Vol, pp 1093-1102. http://dx.doi.org/10.1007/s00253-012-4379-3

SOSA M., PÉREZ-LÓPEZ T., REYES J., CORVO F., CAMACHO-CHAB R., QUINTANA P., AGUILAR D. (2011). Influence of the Marine Environment on Reinforced Concrete Degradation Depending on Exposure Conditions. International Journal of Electrochemical Science, Vol. 6, pp 6300-6318.

SOUCHE J.C., PIOCH S., SAlGUES M., DE WEERDT K., AGOSTINI A., HAYEK M. (2019). De la conception à l'éco-conception des ouvrages maritimes : intégrer la nature au projet d'aménagement maritime. Revue Paralia, Vol. 12, pp 1.1-1.26. http://dx.doi.org/10.5150/revue-paralia.2019.n01

SPERANZA G., GOTTARDI G., PEDERZOLLI C., LUNELLI L., CANTERI R., PASQUARDINI L., CARLI E., LUI A., MANIGLIO D., BRUGNARA M., ANDERLE M. (2004). Role of chemical interactions in bacterial adhesion to polymer surfaces. Biomaterials, Vol. 25, pp 2029-2037. http://dx.doi.org/10.1016/j.biomaterials.2003.08.061

TANG H., WANG A., LIANG X., CAO T., SALLEY S.O., MCALLISTER J.P., NG K.Y.S. (2006). Effect of surface proteins on Staphylococcus Epidermidis adhesion and colonization on silicone. Colloids and Surfaces B: Biointerfaces, Vol. 51, pp 16-24. https://doi.org/10.1016/i.colsurfb.2006.04.011

TEUGHELS W., ASSCHE N.V., SLIEPEN I., QUIRYNEN M. (2006). Effect of material characteristics and/or surface topography on biofilm development. Clinical Oral Implants Research, Vol. 17, pp 68-81. https://doi.org/10.1111/j.1600-0501.2006.01353.x

TRAN T.H., GOVIN A., GUYONNET R., GROSSEAU P., LORS C., DAMIDOT D., et al. (2014). Influence of the intrinsic characteristics of mortars on their biofouling by pigmented organisms: Comparison between laboratory and field-scale experiments. International Biodeterioration and Biodegradation, Vol. 86, pp 334-342. https://doi.org/10.1016/j.ibiod.2013.10.005

VICHOT A., OLLIVIER J. (2008). La durabilité des bétons. École française du béton Paris : Presses de l'École nationale des ponts et chaussées, $844 \mathrm{p}$.

VISHWAKARMA V., GEORGE R.P., RAMACHANDRAN D., ANANDKUMAR B., MUDALI U.K. (2014). Studies of detailed Biofilm characterization on fly ash concrete in comparison with normal and superplasticizer concrete in seawater environments. Environmental Technology, Vol. 35, pp 42-51. https://doi.org/10.1080/09593330.2013.808249 
WHITMAN W.B., COLEMAN D.C., WIEBE W.J. (1998). Prokaryotes: The unseen majority. Proceedings of the National Academy of Sciences, Vol. 95, pp 6578-6583. https://doi.org/10.1073/pnas.95.12.6578

WOOD T.L., GUHA R., TANG L., GEITNER M., KUMAR M., WOOD T.K. (2016). Living biofouling-resistant membranes as a model for the beneficial use of engineered biofilms. PNAS, Vol. 113, pp E2802-E2811. https://doi.org/10.1073/pnas.1521731113 YUAN H., DANGLA P., CHATELLIER P., CHAUSSADENT T. (2015). Degradation modeling of concrete submitted to biogenic acid attack. Cement and Concrete Research, Vol. 70, pp 29-38. https://doi.org/10.1016/j.cemconres.2015.01.002 